\title{
Composición de ácidos grasos de los caracoles marinos Phyllonotus pomum y Chicoreus brevifrons (Gastropoda: Muricidae)
}

\author{
Haydelba D’Armas ${ }^{1 *}$, Dayanis Yáñez ${ }^{1}$, Dilia Reyes ${ }^{1} \&$ Gabriel Salazar $^{2}$ \\ 1. Universidad de Oriente, Núcleo de Sucre. Departamento de Química, Escuela de Ciencias, Cumaná, Estado Sucre \\ 1601, Venezuela; htrinidad86@hotmail.com \\ 2. Instituto Universitario de Tecnología, Cumaná, Estado Sucre 1601, Venezuela. \\ * Correspondencia.
}

Recibido 10-VII-2009. Corregido 25-X-2009. Aceptado 27-XI-2009.

\begin{abstract}
Fatty acids composition of the marine snails Phyllonotus pomum and Chicoreus brevifrons (Muricidae). Muricid species of P. pomum and C. brevifrons are of economic importance in the Caribbean. This study includes a comparative evaluation of fatty acid content in the total lipid composition of Phyllonotus pomum and Chicoreus brevifrons. Snail samples were collected during the rainy, dry and transition seasons, in Punta Arena, Sucre (Venezuela). Total lipids were extracted and the specific fatty acid contents were analyzed by gas chromatography. Lipid concentrations varied between 0.87 and $1.85 \%$, with minimum and maximum values corresponding to C. brevifrons collected during rainy and dry seasons, respectively. In the case of total lipids, a high concentration of unsaturated fatty acids (57.21-70.05\%) was observed followed by saturated fatty acids (20.33-31.94\%), during all seasons. The polyunsaturated occurred in higher proportion among the unsaturated fatty acids, except for $P$. pomum which showed higher proportion of monounsaturated fatty acids $(38.95 \%)$ during the transition season. The prevailing fatty acids were: C14:0, C16:0, C18:0, C20:1, C22:1 $\omega-11, \mathrm{C} 22: 1 \omega-9$, $\mathrm{C} 18: 3 \omega-3, \mathrm{C} 20: 5 \omega-3$ and $\mathrm{C} 22: 6 \omega-3$, among which docosahexaenoic acid was the predominant polyunsaturated fatty acid, showing values between 4.62 and $33.11 \%$. The presence of high concentrations of polyunsaturated fatty acids found in P. Pomum and C. brevifrons allow their recommendation for human consumption with appropriate resource utilization. Rev. Biol. Trop. 58 (2): 645-654. Epub 2010 June 02.
\end{abstract}

Key words: fatty acids, lipids, Phyllonotus pomum, Chicoreus brevifrons, snails.

Los gasterópodos más abundantes en la costa nororiental de Venezuela, son el Chicoreus brevifrons (arrechón, chirigua, chivato, puyuo) y C. (Phyllonotus) pomum (burro, murex manzana), ambos pertenecientes a la familia Muricidae. Dichas especies de caracol son carnívoras y necrófagas, el hábitat común son aguas someras sobre sustratos duros en áreas de manglar, se distribuyen ampliamente en el Atlántico occidental, desde el sur de Florida hasta la costa norte de Brasil (Ablanque \& Borrero 1995, Gómez 1999).

$C$. brevifrons y $P$. pomum tienen importancia económica con potencial de cultivo. La mayoría de los estudios en dichas especies se han basado en aspectos biológicos y bioecológicos (Itriago 1977). En Venezuela, se han realizado algunos trabajos sobre la composición química de varios tipos de moluscos marinos y entre las especies estudiadas, se encuentran los mejillones Perna perna (Gómez 1999, Benítez \& Okuda 1971) y Perna viridis (Koftayan 2003), el caracol Strombus gigas (Malavé 1982) y las ostras Crassostrea virginica (Rosario 1973, Serrano 1982) y Crassostrea rhizophorae (Albornoz 1984, Bonilla et al. 1969, Bonilla 1975). Dichos estudios han puesto en evidencia una fuente lipídica y proteica rica; así como también de ácidos grasos poliinsaturados (AGPI), tal como se reflejara en un estudio 
reciente de la ostra de mangle $C$. rhizophorae (Milano 2003), donde se encontraron altas concentraciones de AGPI del tipo $\omega-3$, particularmente, los ácidos de cadena larga 22:6 $\omega-3$ y 20:5 $\omega-3$, los cuales son fundamentales en la formación de la estructura y la funcionalidad del sistema nervioso y visual de los humanos (Valenzuela \& Nieto 2003), y además juegan un papel muy importante en el tratamiento de ciertas afecciones humanas como las enfermedades cardiovasculares (Leaf 1990).

Todo lo descrito, ha conducido a que actualmente, los análisis de composición química de especies marinas para consumo humano, tengan mayor importancia, ya que este tipo de estudio permite conocer las propiedades alimenticias y energéticas que presentan estos organismos; suministrando información que puede aplicarse con fines terapéuticos y nutricionales. Por ello en la presente investigación se evaluó el perfil de ácidos grasos del contenido lipídico en $P$. pomum y C. brevifrons provenientes de Punta Arena, Sucre, Venezuela, a fin de informar a la población interesada acerca de las propiedades nutricionales que ofrece la ingesta de dichos caracoles marinos y también la de contribuir al desarrollo de la acuicultura de estos moluscos, que en muchas regiones de Venezuela representan un sustento económico $\mathrm{y}$ alimenticio, por ser especies prolíficas y comestibles.

\section{MATERIALES Y MÉTODOS}

Área de estudio y muestreo: La recolección de las muestras se realizó en localidad de Punta Arena, Península de Araya (Cruz Salmerón Acosta, Sucre, Venezuela) en la entrada al Golfo de Cariaco en la parte norte, frente a las costas de Cumaná (10 $30^{\circ}-10^{\circ} 32^{\prime}$ N y 6412'6413' W) (Malavé 1982).

Las especies $P$. pomum y $C$. brevifrons fueron recolectadas manualmente con equipo autónomo de buceo, entre 3 y $25 \mathrm{~m}$ de profundidad, en tres épocas (sequía, lluvia y transición) comprendidas entre octubre 2005 y agosto 2006. Se tomaron 15 caracoles por especie; los cuales se lavaron y refrigeraron en un contenedor para su traslado al laboratorio. Posteriormente, a cada espécimen, se le extrajo la masa blanda (organismo completo y sin concha), misma que se lavó con suficiente agua desionizada para eliminar la mayor cantidad de sales, iones y fitoplancton y luego se congelaron a $-5^{\circ} \mathrm{C}$ para su posterior análisis.

Extracción de lípidos totales: Los lípidos totales se extrajeron por la técnica de Overturf \& Dryer (1967), después de la trituración y maceración del paquete de tejidos en un molino eléctrico. Posteriormente, se tomaron porciones de $1.5 \mathrm{~g}$ del tejido macerado por $30 \mathrm{ml}$ de una mezcla de cloroformo:metanol (2:1). Se agregó como antioxidante butirato de hidroxitolueno (BHT). El tejido con la mezcla de solventes fue sometido a agitación continua (100-500rpm) por una hora, se filtró dos vces en papel de filtro (Whatman $\mathrm{N}^{\circ} 3$ tipo $\mathrm{GF} / \mathrm{C}$ ) para garantizar una extracción eficaz y completa de los lípidos.

El filtrado total se llevó a un embudo de separación, se agregó $\mathrm{NaCl}$ al $0.05 \mathrm{~mol} / 1$, aproximadamente en un tercio del volumen del filtrado, se agitó varias veces y se dejó reposar en refrigeración aproximadamente 16 horas para su separación en solución bifásica. Posteriormente, la fase orgánica con los lípidos se separó y se le añadió $\mathrm{Na}_{2} \mathrm{SO}_{4}$ anhidro luego se filtró a gravedad y la solución se concentró a presión reducida. Después, al extracto lipídico obtenido se le burbujeó nitrógeno gaseoso hasta sequedad y se refrigeró. La cuantificación de los lípidos totales se realizó por gravimetría. El porcentaje de lípidos se determinó mediante la relación [(masa del extracto lipídico/masa del tejido)x100]. Este procedimiento se realizó por triplicado para las muestras en cada muestreo realizado.

Los datos del contenido de lípidos fueron analizados por un análisis de varianza de una vía (ANDEVA). Los análisis estadísticos fueron ejecutados con el paquete estadístico Statgraphics Plus 4.1 con nivel de significancia de $p<0.05$ y al haber diferencias significativas los datos fueron comparados con la prueba $a$ posteriori LSD. 
Esterificación de los ácidos grasos: $\mathrm{La}$ conversión de los ácidos grasos presentes en los lípidos totales a ésteres metílicos se realizó por el método de Brokerchoff (Coristie 1973). Para ello, se tomó de 20 a 40mg de muestra lipídica y se disolvió en $0.5 \mathrm{ml}$ de éter dietílico. Seguidamente, se agregó $1 \mathrm{ml}$ de $\mathrm{KOH} 0.5 \mathrm{~mol} / 1$ en metanol (seco), se agitó vigorosamente y se dejó en reposo durante $15 \mathrm{~min}$. Luego se agregó $1 \mathrm{ml}$ de $\mathrm{HCl} 1 \mathrm{~mol} / 1$ y el producto de la reacción se extrajo con $3.0 \mathrm{ml}$ de éter de petróleo, en tres porciones de $1.0 \mathrm{ml}$. Los ésteres metílicos de los ácidos grasos obtenidos, se reunieron y el volumen total del solvente se evaporó con nitrógeno gaseoso.

Identificación y cuantificación de los ácidos grasos: La composición porcentual de los ácidos grasos en los extractos de lípidos totales se determinó mediante cromatografía de gas-líquido (CG). Los ésteres metílicos de los ácidos grasos correspondientes a cada muestra se analizaron en un cromatógrafo de gas-líquido (Agilent 4890 D), acoplado a un detector de ionización de llama (FID) y una columna HPINNOXWAX $30 \mathrm{~m}$ de $0.32 \mathrm{~mm}$ ID (diámetro interno) y $0.5 \mu \mathrm{m}$ de espesor de fase líquida. Se utilizó nitrógeno como gas de arrastre a un flujo de $1.8 \mathrm{ml} / \mathrm{min}$. La separación se realizó en las siguientes condiciones: Temperatura del inyector: $300^{\circ} \mathrm{C}$, temperatura del detector: $300^{\circ} \mathrm{C}$ y temperatura de la columna: $200^{\circ} \mathrm{C}$.
El área de los picos se determinó con un sistema de adquisición de data HP3398AGC CHEMSTATION acoplado al cromatógrafo. La identificación de ácidos grasos se realizó mediante la comparación de los tiempos de retención, determinados con los patrones comerciales de ésteres metílicos (Farnworth et al. 1982).

Los resultados se expresaron en porcentaje del total de ácidos grasos presentes en los extractos lipídicos de los caracoles para cada muestreo.

\section{RESULTADOS}

Determinación de los lípidos totales: En el caracol C. brevifrons el contenido de lípidos totales osciló entre 0.87 y $1.85 \%$, correspondiendo el valor mínimo y el máximo de las muestras recolectadas en época de sequía y lluvia respectivamente (Cuadro 1). El análisis de varianza aplicado a estos valores indicó la existencia de diferencias significativas $(\mathrm{p}<0.05)$ a un intervalo de confianza del $95 \%$, lo cual se constató a través de una prueba a posteriori LSD (Cuadro 1), donde se observó la formación de tres grupos homogéneos (GH:X) conformados por las muestras de cada uno de los muestreos realizados. En $P$. pomum, el análisis de varianza evidenció la existencia de diferencias significativas en el contenido lipídico de esta especie (0.93-1.64\%), con la formación de tres grupos independientes constituidos por las

CUADRO 1

Porcentajes de lípidos totales de los moluscos C. brevifrons y P. pomum

TABLE 1

Percentages of total lipids of $\mathrm{C}$. brevifrons and $\mathrm{P}$. pomum mollusks

\begin{tabular}{lllcc} 
Especie & Época de muestreo & Intervalo & $(\overline{\mathrm{X}} \pm \mathrm{S})$ & $(\mathrm{GH})$ \\
C. brevifrons & Transición & $1.08-1.58$ & $1.33 \pm 0.25$ & $\mathrm{X}$ \\
& Sequía & $0.76-0.99$ & $0.87 \pm 0.12$ & $\mathrm{X}$ \\
\multirow{3}{*}{ pomum } & Lluvia & $1.84-1.85$ & $1.85 \pm 0.01$ & $\mathrm{X}$ \\
& Transición & $0.90-0.97$ & $0.93 \pm 0.04$ & $\mathrm{X}$ \\
& Sequía & $1.54-1.83$ & $1.64 \pm 0.16$ & $\mathrm{X}$ \\
& Lluvia & $1.29-1.33$ & $1.31 \pm 0.02$ & $\mathrm{X}$
\end{tabular}

$\bar{X}$ : Media; S: Desviación estándar; GH: Grupo homogéneo (LSD). 
muestras colectadas en los periodos de transición, sequía y lluvia respectivamente, hallándose la mayor concentración lipídica en el grupo conformado por las muestras de sequía.

Análisis de los ácidos grasos: La composición de ácidos grasos de los lípidos totales para $C$. brevifrons y $P$. pomum esta entre 20.33 y $33.39 \%$, correspondiendo ambos valores al caracol C. brevifrons, colectado en el período de transición y lluvia, respectivamente (Cuadro 2 y 3). Estos ácidos grasos están constituidos mayoritariamente por los ácidos mirístico (C14:0), palmítico (C16:0), esteárico $(\mathrm{C} 18: 0)$ y lignocérico (C24:0).

Por otra parte, los dos caracoles mostraron altos contenidos de ácidos grasos insaturados (57.21-70.05\%), estos estuvieron caracterizados

CUADRO 2

Composición porcentual de los ácidos grasos en los lipidos totales del C. brevifrons

TABLE 2

Composition of fatty acids from total lipids of C. brevifrons

\begin{tabular}{|c|c|c|c|}
\hline \multirow{2}{*}{ Ácidos grasos } & \multicolumn{3}{|c|}{ \% de ácidos grasos (Área) } \\
\hline & Transición & Sequía & Lluvia \\
\hline C 9:0 & - & 0.11 & - \\
\hline C 10:0 & - & 0.07 & 0.47 \\
\hline C 11:0 & - & 0.67 & - \\
\hline C 13:0 & - & 0.27 & - \\
\hline C $14: 0$ & 1.76 & 3.23 & 3.80 \\
\hline C $16: 0$ & 4.84 & 10.31 & 17.27 \\
\hline C 18:0 & 4.78 & 8.56 & 11.12 \\
\hline C 20:0 & 2.71 & - & - \\
\hline C 21:0 & 1.79 & - & - \\
\hline C $24: 0$ & 4.45 & 5.60 & 0.73 \\
\hline Total AGS & 20.33 & 28.82 & 33.39 \\
\hline C $16: 1$ & 1.10 & 2.69 & 3.08 \\
\hline 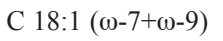 & 2.45 & 4.21 & 1.00 \\
\hline C $20: 1$ & 6.71 & 7.12 & - \\
\hline C $22: 1 \omega-9$ & 3,27 & 5.22 & 2.99 \\
\hline C $22: 1 \omega-11$ & 3.13 & 7.30 & 14.33 \\
\hline C 24:1 T.I & 7.61 & 5.06 & - \\
\hline Total AGMI & 24.27 & 31.60 & 21.40 \\
\hline C 16: 2 & 0.95 & 1.51 & 2.13 \\
\hline C 18: 2 & 1.79 & 2.52 & 1.97 \\
\hline C 18: 3 & 1.98 & 3.60 & 1.96 \\
\hline C 18: 4 & 4.33 & 0.99 & 9.08 \\
\hline C 20: 2 & 4.13 & 0.63 & 2.49 \\
\hline C 20: 3 & 2.20 & 5.29 & - \\
\hline C $20: 5 \omega-3$ & 1.46 & 2.95 & 12.48 \\
\hline C $22: 6 \omega-3$ & 28.94 & 14.77 & 5.70 \\
\hline Total AGPI & 45.78 & 32.26 & 35.81 \\
\hline Total AGI & 70.05 & 64.86 & 57.21 \\
\hline Total AGNI & 9.62 & 7.32 & 9.40 \\
\hline
\end{tabular}

AGS: Ácidos grasos saturados; AGMI: Ácidos grasos monoinsaturados; AGPI: Ácidos grasos poliinsaturados; AGI: Ácidos grasos insaturados; AGNI: Ácidos grasos no identificados; $\omega=$ n; TI: Tentativamente identificado como; (-): No se encontró dicho ácido graso. 
CUADRO 3

Composición porcentual de los ácidos grasos en los lípidos totales del P. pomum

TABLE 3

Composition of fatty acids from total lipids of $\mathrm{P}$. pomum

\begin{tabular}{|c|c|c|c|}
\hline \multirow{2}{*}{ Ácidos grasos } & \multicolumn{3}{|c|}{ \% de ácidos grasos (Área) } \\
\hline & Transición & Sequía & Lluvia \\
\hline C 9:0 & 0.08 & 0.07 & - \\
\hline C 10:0 & 0.06 & 0.22 & 0.60 \\
\hline C 11:0 & 1.28 & 0.38 & - \\
\hline C 13:0 & - & 0.19 & - \\
\hline C $14: 0$ & 2.26 & 2.21 & 2.29 \\
\hline C $16: 0$ & 4.11 & 7.96 & 9.06 \\
\hline C $18: 0$ & 5.70 & 6.33 & 9.25 \\
\hline C 21:0 & 2.08 & 4.18 & - \\
\hline C $24: 0$ & 6.48 & 10.40 & 2.74 \\
\hline Total AGS & 22.05 & 31.94 & 23.94 \\
\hline C $16: 1$ & 1.59 & 2.14 & 1.77 \\
\hline C $18: 1(\omega-7+\omega-9)$ & 5.25 & 3.68 & 3.21 \\
\hline C $20: 1$ & 10.44 & 6.52 & 2.82 \\
\hline C $22: 1 \omega-9$ & 7.26 & 4.97 & 4.20 \\
\hline C $22: 1 \omega-11$ & 7.90 & 5.35 & 16.92 \\
\hline C 24:1 T.I & 6.51 & 5.02 & - \\
\hline Total AGMI & 38.95 & 27.68 & 28.92 \\
\hline C 16: 2 & 0.48 & 1.19 & 1.59 \\
\hline C 18: 2 & 2.04 & 1.46 & 1.81 \\
\hline C 18: 3 & 3.33 & 2.09 & 2.29 \\
\hline C 18: 4 & 2.29 & - & 8.70 \\
\hline C 20: 2 & 1.43 & 0.65 & - \\
\hline C 20: 3 & 8.08 & 4.15 & 1.66 \\
\hline C $20: 5 \omega-3$ & 1.87 & 2.90 & 11.96 \\
\hline C $22: 6 \omega-3$ & 7.36 & 21.63 & 6.74 \\
\hline Total AGPI & 26.26 & 34.07 & 35.37 \\
\hline Total AGI & 65.21 & 61.75 & 64.29 \\
\hline Total AGNI & 12.74 & 6.31 & 11.77 \\
\hline
\end{tabular}

AGS: Ácidos grasos saturados; AGMI: Ácidos grasos monoinsaturados; AGPI: Ácidos grasos poliinsaturados; AGI: Ácidos grasos insaturados; AGNI: Ácidos grasos no identificados; $\omega=$ n; TI: Tentativamente identificado como; (-): No se encontró dicho ácido graso.

por ser mayormente de cadenas largas de 18, 20 y 22 átomos de carbono. Dentro de los mismos, los ácidos grasos poliinsaturados fueron los predominantes en ambas especies, en todas las épocas, exceptuando el período de transición donde el extracto lipídico del caracol $P$. pomum mostró un predominio cuantitativo de los ácidos grasos monoinsaturados (AGMI, 38.95\%).
Losácidos grasosmonoinsaturados(AGMI) oscilaron entre $21.40 \%$ (lluvia) y $38.95 \%$ (transición), correspondiendo el menor y mayor valor a $C$. brevifrons y $P$. pomum, respectivamente. Estos se encontraron representados por los ácidos: palmitoleico (C16:1), octadecenoico (vaccénico $(\omega-7)$ y oleico $(\omega-9))$, gadoleico (C20:1), erúcico (C22:1 $\omega-9)$, cetoleico (C22:1 
$\omega$-11) y nervónico (C24:1), este último hallado solo en las épocas de transición y sequía. Los mayores porcentajes de distribución se observaron para el ácido cetoleico (1.83-16.92\%), principalmente en la época de lluvia.

Por su parte, los ácidos grasos poliinsaturados (AGPI) fluctuaron entre 26.26 y $45.78 \%$ correspondiendo el valor mínimo y máximo a $P$. pomun y C. brevifrons respectivamente, colectados en el periodo de transición. Estos ácidos se encontraron representados principalmente por los ácidos $\omega$-3, octadecatrienoico (C18:3), eicosapentaenoico (C20:5) y docosahexaenoico (C22:6), siendo este último el de valores más elevados (5.70-28.94\%), primordialmente en los períodos de transición y sequía.

\section{DISCUSIÓN}

Las diferencias observadas en el contenido lipídico de las muestras de una misma especie, colectadas en distintas épocas del año, pueden estar relacionadas con las condiciones ambientales de la zona de muestreo al momento de captura (salinidad, temperatura, clorofila $a$, oleaje), la disponibilidad de alimentos, diferencias biológicas como tamaño, sexo y ciclo biológico o desove de la especie entre otros factores (endógenos y/o exógenos), que pudieron influir en la variabilidad estacional de los lípidos (Freites 2002). Con respecto a esto, se ha señalado que la gran mayoría de los invertebrados marinos presentan fluctuaciones en su demanda energética, debido a las variaciones en los procesos fisiológicos naturales, los cuales son regulados de manera compleja por factores endógenos de carácter bioquímico, que a su vez están influenciados por las condiciones externas ambientales (Gharseddiene 1999). En dicho sentido, algunos autores han manifestado que la disponibilidad de alimentos con elevado valor nutritivo es uno de los factores exógenos más importantes que pueden incidir, conjuntamente con diversos factores endógenos, en la variación estacional de ciertos componentes energéticos como los lípidos (Gardner \& Riley 1972).
Considerando los resultados obtenidos, el rango del contenido de lípidos osciló entre $0.87 \%$ y $1.85 \%$ (Cuadro 1). Estos valores están comprendidos en el intervalo de porcentajes reportados en un estudio de los parámetros de frescura de siete moluscos marinos: (Donax $s p$ ("chipichipi"), Tivela mactroides ("guacuco"), Arca zebra ("pepitota"), P. viridis ("mejillón verde"), Pinctada imbricata ("ostra perla"), Loligo pler ("calamar") y Octupus vulgaris ("pulpo"), donde se hallaron contenidos de grasas entre 1.08 y $3.03 \%$, siendo el "chipichipi", el "mejillón verde" y la "pepitota" los de mayor contenido lipídico (Cabello et al. 2004).

La máxima concentración de lípidos $(1.64 \%)$ observada en la especie $P$. pomum en época de sequía puede estar relacionada con la alta disponibilidad de alimentos que se da durante ese periodo en la zona, debido al fenómeno de surgencia. En los primeros meses del año (febrero-mayo), Punta Arena se ve mayormente afectada por los vientos alisios, que desplazan el agua superficial y producen el ascenso de las aguas subsuperficiales hacia la superficie. Las aguas surgidas son más frías y ricas en sales nutritivas que fertilizan el mar y favorecen la abundancia de plantas y animales microscópicos, que constituyen el alimento de larvas y jóvenes de muchas especies marinas (Gómez 1999, Jordán 1997).

La homogeneidad observada en el contenido lipídico de $C$. brevifrons y $P$. pomum pudiera estar relacionada con el hecho que ambas especies son gasterópodos pertenecientes a la misma familia, Muricidae. Los valores lipídicos (promedio de los tres muestreos) son altos en comparación con los hallados en la carne comestible de otros gasterópodos marinos tales como (Brito \& Aldana 1998): Busycon carica $(0.09 \%)$, Pleuropioca gigantea $(0.27 \%)$, Xancus angulatus $(0.06 \%)$, Strombus costatus $(0.83 \%)$ y $S$. galeatus $(0.26 \%)$. Sin embargo, son relativamente bajos en comparación con $S$. gigas $(2.75 \%)$ en un estudio realizado en Punta Arena (Malavé 1982).

Los ácidos grasos saturados están constituidos mayoritariamente por los ácidos mirístico (C14:0), palmítico (C16:0), esteárico (C18:0) 
y lignocérico (C24:0). Cabe destacar que los mayores porcentajes de distribución correspondieron a los ácidos C16:0 (4.11-31.32\%) y C18:0 (4.78-13.20\%), resultados que coinciden con estudios previos en moluscos marinos (Christie 1990, Freites 2002), donde se ha señalado a los ácidos palmítico y esteárico como los ácidos grasos saturados más abundantes en los lípidos totales. En cuanto al prevalecimiento del ácido C16:0, es de hacer notar que éste es el principal AGS constituyente de los fosfolípidos. Las elevadas concentraciones de este ácido graso, por encima del $10 \%$, que mostró C. brevifrons en las épocas de sequía (10.31\%) y lluvia (17.27\%), son comparables con las reportadas en la literatura (Koftayan 2003) para el mejillón Perna viridis en Río Caribe y La Esmeralda (10.40 y $33.72 \%$ ), estado Sucre.

Para ambas especies, los ácidos grasos poliinsaturados (AGPI) se encontraron representados principalmente por los ácidos $\omega-3$, octadecatrienoico (C18:3), eicosapentaenoico (C20:5) y docosahexaenoico (C22:6), siendo este último el más elevado (5.70-28.94\%), primordialmente en los períodos de transición y sequía. Este señalamiento es muy importante, ya que según numerosas investigaciones, los ácidos grasos $\omega$-3 y sus metabolitos proporcionan múltiples efectos benéficos en la salud, tanto en el tratamiento como en la prevención de numerosas enfermedades, tales como: padecimientos de corazón por su efecto hipocolesterolémico e hipotensivo, en forma directa y como coadyuvante en el tratamiento de artritis y cáncer por su efecto antiinflamatorio; la diabetes; enfermedades renales, ayudando a la normalización del metabolismo de lípidos; en desórdenes mentales, como la pérdida de memoria, pues son uno de los componentes principales de las células del cerebro; en el tratamiento de la depresión y en el mejoramiento del desarrollo pre y post natal (Kinsella 1986, Romero et al. 2002).

Es importante resaltar que las concentraciones obtenidas para el ácido docosahexaenoico en este estudio, sobrepasan a las registradas previamente (Koftayan 2003, Milano 2003), para los moluscos C. rhizophorae (1.5-1.7\%) y Perna viridis (0.19-8.07\%). Con respecto a estos resultados, se ha señalado que un alto porcentaje de un ácido graso con funciones estructurales como el C22:6, podría ser un mecanismo de adaptación del animal a condiciones extremas del medio que lo rodea (Freites 2002).

Por otra parte, algunos investigadores refieren que la presencia del ácido docosahexaenoico, así como también de otros AGPI de cadena larga, tales como los ácidos C20:5 y C22:5, además de ser una de las características más importantes de los lípidos marinos, contribuye al carácter tradicionalmente inestable de este tipo de materia grasa (Romero et al. 2002, Castro-González et al. 2004).

Los ácidos grasos poliinsaturados del tipo $\omega-3$ son esenciales para los animales marinos, debido a que los mismos no pueden ser sintetizados y se deben adquirir mediante la dieta diaria; se ha considerado que son obtenidos del fitoplancton, el cual es la mayor fuente de alimento de muchos organismos marinos y que contiene a su vez, una alta proporción de AGPI de más de 20 átomos de carbono (Andarcia 1997, Romero et al. 2002, Castro-González et al. 2004).

En los lípidos totales de C. brevifrons y $P$. pomum hay un predominio de los ácidos grasos insaturados sobre los saturados en todas las épocas de muestreo, resultados que concuerdan con estudios previos sobre la composición de ácidos grasos de invertebrados marinos (Milano 2003), en los cuales predominaron las grasas insaturadas en los lípidos de dichos organismos.

En cuanto a las variaciones estacionales que mostraron los ácidos grasos, se puede observar que los mayores porcentajes de distribución de los ácidos grasos poliinsaturados se registraron en la época de transición para C. brevifrons y en la época de sequía para $P$. pomum, periodos donde estas especies presentaron altas concentraciones lipídicas. Con respecto a esto, en un estudio del perfil lipídico de 25 peces marinos, se observó que el contenido de ácidos grasos $\omega$-3 fue mayor que el de ácidos grasos $\omega-6$ independientemente del 
contenido de grasa en la carne, con una tendencia a elevar el contenido de ambos tipos de ácidos grasos conforme aumenta el porcentaje de grasa muscular (Castro-González et al. 2004).

Estudios realizados en invertebrados marinos han relacionado un alto porcentaje de distribución de los AGPI como en las especies estudiadas (mayormente en sequía y transición), con los períodos de madurez sexual de las especies, debido posiblemente a un acondicionamiento bioquímico de las células germinales para cumplir sus funciones gametogénicas. Mientras que un marcado descenso de los AGPI conjuntamente con un mayor contenido de AGS como en C. brevifrons (época de lluvia), se ha relacionado con los periodos de desove sexual, y se ha señalado como una posible causa de la degradación de los ácidos grasos poliinsaturados para la formación de eicosanoides, compuestos con una gran actividad fisiológica y farmacológica, como el caso de las prostaglandinas, las cuales tienen una participación importante en el crecimiento y reproducción de dichas especies (Malavé 1995, Andarcia 1997).

Por otra parte, algunos autores sugieren que las variaciones en los AGPI pueden deberse a que al ser componentes principales de los fosfolípidos y formar parte de las membranas, pueden ser usados como combustibles metabólicos. Además, señalan que la variabilidad de los ácidos grasos puede estar relacionada con los hábitos alimenticios de las especies marinas, así como con la disponibilidad de alimentos (fitoplancton) del medio (Malavé 1995, Gharseddiene 1999). En este sentido, otros investigadores (Romero et al. 2002) consideran que los factores ambientales como la dieta, la época de captura y la temperatura del agua, además de algunas diferencias biológicas tales como edad, sexo y tamaño de las especies, afectan la composición de ácidos grasos en las mismas.

Las especies estudiadas presentaron altos contenidos de ácidos grasos insaturados del tipo $\omega-3$, principalmente del ácido docosahexaenoico (5.70-28.94\%), posiblemente su consumo frecuente sea una rica fuente de ácidos grasos esenciales para el organismo, lo cual pudiera resultar beneficioso para la salud.

\section{AGRADECIMENTOS}

Los autores agradecen a Iván López, por la recolección e identificación de las especies; y al Consejo de Investigación y al Departamento de Química de la Universidad de Oriente, Venezuela, por el financiamiento de esta investigación.

\section{RESUMEN}

Se comparó el contenido de ácidos grasos en los lípidos totales de los caracoles Phyllonotus pomum y Chicoreus brevifrons, recolectados en tres diferentes épocas del año en Punta Arena, estado Sucre, Venezuela. Las concentraciones lipídicas oscilaron entre 0.87 y $1.85 \%$, correspondiendo el valor mínimo y el máximo a $C$. brevifrons colectado en lluvia y sequía, respectivamente. La esterificación de los ácidos grasos presentes en estos lípidos seguida de la cromatografía de gases permitió la caracterización y cuantificación de los ácidos grasos. En los lípidos totales de las dos especies, en todas las épocas, se observaron elevadas concentraciones de ácidos grasos insaturados (57.21-70.05\%) seguido de los saturados (20.33-31.94\%). Entre los ácidos grasos insaturados, predominaron los de tipo poliinsaturados, exceptuándose el extracto lipídico de $P$. pomum en época de transición donde las grasas monoinsaturadas fueron las mayoritarias $(38.95 \%)$. Los ácidos grasos que prevalecieron fueron: C14:0, C16:0, C18:0, C20:1, C22:1 $\omega-11$, C22:1 $\omega-9$, C18:3 $\omega-3$, C20:5 $\omega-3$ у el C22:6 $\omega-3$, siendo este último el predominante entre los ácidos grasos poliinsaturados, presentando porcentajes de distribución comprendidos entre 4.62 y $33.11 \%$. Debido a las altas concentraciones de ácidos grasos poliinsaturados encontradas en ambos caracoles, se recomienda su consumo para la alimentación de los seres humanos.

Palabras clave: ácidos grasos, caracoles, Chicoreus brevifrons, Phyllonotus pomum, lípidos.

\section{REFERENCIAS}

Ablanque, F. \& F. Borrero. 1995. Influencia de la selectividad por sustrato de fijación de la Pteria colymbus (Bivalvia: Pteriidae) en la región de Santa Marta, Caribe colombiano. VI COLACMAR 1: 11.

Albornoz, L. 1984. Variación quincenal de la composición química de la ostra Crassostrea rhizophorae (Guilding) natural y cultivada en la Laguna de La 
Restinga, Isla de Margarita. Trabajo de pregrado. Universidad de Oriente, Núcleo de Sucre, Cumaná, Venezuela.

Andarcia, W. 1997. Caracterización y cuantificación de lípidos en el hígado, gónadas femeninas y tejido muscular de la especie marina Thalassophryne maculosa (sapo cano). Trabajo de pregrado, Universidad de Oriente, Núcleo de Sucre, Cumaná, Venezuela.

Benítez, J. \& T. Okuda. 1971. Variación estacional de la composición química del mejillón Perna perna natural. Bol. Inst. Oceanogr. Univ. Oriente 10: 3-8.

Bonilla, J., J. Benitez \& T. Okuda. 1969. Variación estacional de la composición química del ostión $C$. rhizophorae (Guilding) en Laguna Grande y la Bahía de Mochima. Bol. Inst. Oceanogr. Univ. Oriente 8: 46-52.

Bonilla, J. 1975. Variación mensual de la composición química del ostión de mangle en la Laguna Las Maritas (Venezuela). Bol. Inst. Oceanogr. Univ. Oriente 14: 117-127.

Brito, N. \& D. Aldana. 1998. Variación estacional de lípidos en varios tejidos del cambute Strombus gigas (Mesogastropoda: Strombidae), en Quintana Roo, México. Rev. Biol. Trop. 46: 655-660.

Cabello, A., R. Villarroel, B. Figuera, M. Ramos, Y. Márquez \& O. Vallenilla. 2004. Parámetros de frescura de moluscos. Revista Científica 14: 457-466.

Castro-González, M., A. Ojeda, J. Silencio, L. Cassis, H. Ledesma \& F. Pérez. 2004. Perfil lipídico de 25 pescados marinos mexicanos con especial énfasis en sus ácidos grasos n-3 como componentes nutracéuticos. Alan 54: 328-336.

Christie, W. 1990. Gas chromatography and lipids: A practical guide. The Oily, Bridgwater, Inglaterra.

Coristie, W. 1973. Lipids analysis. Pergamon, Nueva York, EEUU.

Farnworth, E., B. Thompson \& J. Kramer. 1982. Quantitative determination of neutral lipids on chromarods. J. Chromatogr. 264: 463-747.

Freites, L. 2002. Composición bioquímica, clases de lípidos y ácidos grasos del mejillón Mytilus galloprovincialis (Lamarck, 1819), en cultivo. Influencia del origen de las semillas y de los parámetros ambientales. Tesis Doctoral, Universidad de Santiago de Compostela, Santiago de Compostela, España.

Gharseddiene, S. 1999. Bioecología del isótopo litoral Tylos wegeneri (crustácea: isopoda) en Guayacán, estado Sucre, Venezuela. Tesis Maestría, Universidad de Oriente, Núcleo de Sucre, Cumaná, Venezuela.

Gardner, D. \& J. Riley. 1972. Seasonal variations in the component fatty acid distributions of the lipids of Balanus balanoides. J. Mar. Biol. Ass. U.K. 52: 839-845.

Gómez, A. 1999. Los recursos marinos renovables del Estado Nueva Esparta Venezuela. Biología y pesca de las especies comerciales. Tomo I. Organización Gráficas Capriles, Caracas, Venezuela.

Itriago, M. 1977. Estudio citogenético y anatómico de las especies Chicoreus brevifrons (Lamarck, 1822) y Chicoreus pomum (Gmelin, 1791) (Neogastropoda: Muricidae). Trabajo de pregrado, Universidad de Oriente, Núcleo de Sucre, Cumaná, Venezuela.

Jordán, N. 1997. Cambios estacionales en la composición bioquímica del seston en las localidades de Chacopata (Península de Araya) y Punta Arenas (Golfo de Cariaco), estado Sucre, Venezuela. Trabajo de ascenso, Universidad de Oriente, Cumaná, Venezuela.

Kinsella, J. 1986. Food components with potential therapeutic benefits: the polyunsaturated fatty acids of oils. Food Technol. 35: 94-95.

Koftayan, T. 2003. Estudio comparativo del contenido lipídico de la especie marina Perna viridis (mejillón verde), presente en el oriente Venezolano y en la costa oeste de la Isla de Trinidad. Trabajo de pregrado, Universidad de Oriente, Cumaná, Venezuela.

Leaf, D. 1990. Ácidos grasos n-3 y enfermedades coronarias. Tribuna médica 73: 280-384.

Malavé, D. 1982. Composición química y contenido de elementos trazas en el botuto Strombus gigas (Linnaeus, 1758). Trabajo de pregrado, Universidad de Oriente, Núcleo de Sucre, Cumaná, Venezuela.

Malavé, M. 1995. Caracterización y cuantificación del contenido lipídico y ácidos grasos presentes en dos especies de erizos de mar: Lytechinus variegatus y Echinometra lucunter. Trabajo de pregrado, Universidad de Oriente, Cumaná, Venezuela.

Milano, J. 2003. Estudio comparativo del contenido lipídico, proteico y de glucógeno en la Crassostrea rhizophorae (ostra de mangle) provenientes de la laguna Chacopata, la Restinga y Caroní Swamp. Trabajo de pregrado, Universidad de Oriente, Cumaná, Venezuela.

Overturf, M. \& R. Dryer. 1967. Experiments in physiology and biochemistry. Academic, Nueva York, EEUU. 
Romero, N., P. Robert, L. Masson \& R. Pineda. 2002. Composición en ácidos grasos y proximal de siete especies de pescado de Isla de Pascua. Alan 50: 304-308.

Rosario, L. 1973. Variación mensual en la Composición química de la ostra Crassostrea virginica (Gmelin, 1970) de la zona de Guariquén. Tesis de pregrado, Universidad de Oriente, Núcleo de Sucre, Cumaná, Venezuela.
Serrano, I. 1982. Variación estacional en la composición química de la ostra Crassostrea virginica (Gmelin, 1970) cultivada en los caños de Guariquen. Tesis de pregrado, Universidad de Oriente, Núcleo de Sucre, Cumaná, Venezuela.

Valenzuela, A. \& S. Nieto. 2003. Ácidos grasos omega-6 y omega-3 en la nutrición perinatal: $\mathrm{Su}$ importancia en el desarrollo del sistema nervioso y visual. Rev. Chil. Pediatr. 74: 149-157. 